\title{
Hyperspectral Image Processing in Internet of Things model using Clustering Algorithm
}

\section{Bindhu V,}

Professor and Head,

Department of Electronics and Communication Engineering, PPG Institute of Technology,

Coimbatore, India.

Email: vbindhuppg@gmail.com

\section{G. Ranganathan,}

Professor and Head,

Department of Electronics and Communication Engineering,

Gnanamani College of Technology,

Namakkal, India.

Email:profranganathang@gmail.com

\begin{abstract}
With the advent of technology, several domains have b on Internet of Things (IoT). The hyper spectral sensors present in earth observation system sends hyper spectral images (HSIs) to the cloud for further processing. Artificial intelligence (AI) models are used to analyse data in edge servers, resulting in a faster response time and reduced cost. Hyperspectral images and other high-dimensional image data may be analysed by using a core AI model called subspace clustering. The existing subspace clustering algorithms are easily affected by noise since they are constructed based on a single model. The representation coefficient matrix connectivity and sparsity is hardly balanced. In this paper, connectivity and sparsity factors are considered while proposing the subspace clustering algorithm with post-process strategy. A non-dominated sorting algorithm is used for that selection of close neighbours that are defined as neighbours with high coefficient and common neighbours. Further, pruning of useless, incorrect or reserved connections based on the coefficients between the close and sample neighbours are performed. Lastly, inter and intra subspace connections are reserved by the post-process strategy. In the field of IoT and image recognition, the conventional techniques are compared with the proposed postprocessing strategies to verify its effectiveness and universality. The clustering accuracy may
\end{abstract}


be improved in the IoT environment while processing the noise data using the proposed strategy as observed in the experimental results.

Keywords: Hyperspectral Images, Internet of Things, Subspace Clustering, Artificial Intelligence, Sorting algorithm

\section{Introduction}

Global positioning system, radio frequency identification technology, sensors and various other devices are involved in the process of data collection in the Internet of Things (IoT) [1]. The connection between multiple things and people are realized by various networks. Various domains like environmental monitoring, recommendation systems, forest protection, smart transportation, healthcare and smart city has several applications that incorporate IoT technologies. Hyperspectral sensors are used for imaging specific regions by using hyperspectral remote sensing technology in earth observation field [2]. The Hyperspectral images (HSIs) generated from these sensors depict the field objects in a detailed manner. This technique substantially improves classification and identification skills. Environmental monitoring, atmospheric environment, fine agriculture, geological mapping, geological exploration and various other fields will widely use HSI technology [3].

The cloud infrastructure is used for transferring and analysing the HSI data in Earth observation systems by using IoT [4]. High energy consumption and latency may be observed in this scenario. The edge servers are deployed with artificial intelligence (AI) models for performing data analysis by leveraging prompt reply and cost reduction. Land-cover classes are assigned to pixels in the HSI data analysis by utilising AI classification. [5]. Supervised and unsupervised classification are the categories under which the HSI is classified. Several applications under various domains make use of supervised classification. In order to train in advance, it is essential to have several labelled samples [6]. In edge servers, this will cause serious cost pressure [7]. However, only the HSI features are considered for performing unsupervised classification [8]. Clustering technique is widely used and extensively studied 
in the unsupervised classification. The high data dimensionality makes the conventional clustering approaches unsuitable for HSI. In high-dimensional information, the subspace structure of low-dimension may be discovered with the subspace clustering that has been fabricated for the high-dimensional information [9]. The fields of computer vision are inclusive of motion segmentation, image segmentation and face clustering, which makes use of this technique. At edge servers, the HSI cluster pixels are deployed with subspace clustering based on AI models [10]. The corresponding subspace is assigned with samples by using subspace clustering [11]. Spatial clustering is used for obtaining the clustering results by initially acquiring the representation coefficient matrix with the help of a subspace clustering framework as represented in figure 1.

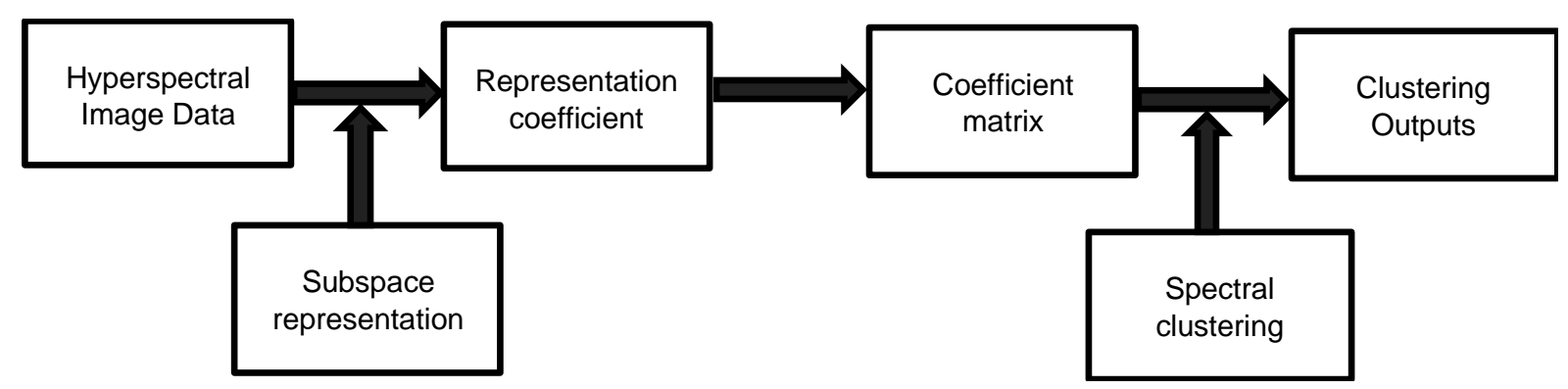

Fig.1. Subspace clustering framework

In IoT domain, the hyperspectral datasets are processed with an improved post-process strategy based on subspace clustering algorithm proposed in this paper. In improved subspace clustering, the representation coefficient matrix parameters like connectivity and sparsity are balanced by using the proposed post-process strategy [12]. The correlations are considered along with the common neighbour numbers, while defining the close neighbours. Nondominated sorting algorithm is used for the selection of close neighbours from the available neighbours in the sample while simultaneously considering both the objectives. The postprocess strategy universality and effectiveness are verified with HSI and traditional picture datasets through experimentation. Four diverse HSI data are used for verification under IoT domain. The original algorithm is compared with various subspace clustering algorithms with the help of pruning technology. The clustering accuracy may be improved in the Earth 
Journal of ISMAC (2021)

Vol.03/ No.02

Pages: 163-175

http://irojournals.com/iroismac/

DOI: https://doi.org/10.36548/jismac.2021.2.008

observation system based on IoT by processing the image data of high dimensionality using the proposed algorithm as observed from the experimental results [13].

\section{Related Works}

In 2009, the subspace clustering was introduced and has been gaining large attention from several fields ever since. Computation of the representation coefficient matrix is the key to subspace clustering. Regularization term and fidelity term are the major components in the matrix computational model. For each sample, a linear expression is sought after by using a fidelity terms to create a dictionary of samples that belong to the same subspace [14]. The block-diagonal structure matrix is satisfied using the regularization term. Different matrices are produced by different norms making it critical to design the regularization term [15]. Mixture, connectivity and sparsity are the three major directions involved in the regularization term design [16]. The sparse representation coefficient matrix is computed with the regularization terms based on $\mathrm{L}_{0}$ and $\mathrm{L}_{1}$. Sparse 1-D based Sparse Subspace Clustering(SSC) is used, where, in a single subspace, a sample is used for representing each data sample forcefully using a regularization term based on $\mathrm{L}_{1}$ norm [17].

Mutually independent subspaces are used for meeting the block-diagonal structure using this solution model. The data subspace properties are revealed by this structure such as a single subspace comprising of samples in a block, and equal number of subspaces and blocks. The Laplacian matrix and its zero eigen values count is constrained which in turn constrains the total blocks in the matrix using Block Diagonal Representation (BDR) [18]. HSI clustering, end-member extraction, and hyperspectral anomaly detection are applied with SSC by certain researchers. Spectral-spatial SSC is the HSI clustering based SSC developed in 2006. KSSCSMP, a spatial max pooling operation is applied in a kernel SSC scheme [19]. SSC algorithm based on spatial distribution preserving and SSC scheme regularized by $\mathrm{L}_{0}$-norm are used for as improving algorithms. An $\mathrm{L}_{0}$-norm based model is proposed in combination with proximal gradient descent method along with SSC [20]. NP-hard problems are often caused by minimization of $\mathrm{L}_{0}$-norm. Nearest subspace neighbour and orthogonal matching pursuit algorithms are proposed to overcome this issue. Heuristic methods are used for obtaining 
non-zero positions using orthogonal matching pursuit based subspace clustering (SSC-OMP). The general pair wise relationship may not be sufficient for expressing the sample coefficients [21]. A disconnected graph of subspace is over-pursued aggressively using the sparse matrix.

\section{Proposed Work}

The data samples are divided into appropriate subspaces for the purpose of subspace clustering. A data matrix $D=\left[D_{1}, D_{2}, \ldots . D_{n}\right] \in \Re^{\text {ds } X n}$ where the set of $n_{i}$ samples belonging to a subspace $S_{i}$ is represented by $D_{i}, d_{s}$ is the sample dimension and $n$ is the total samples in the data. The position or noise in the sample is caused by useless or incorrect connections in real time applications. When compared to the subspace intrinsic dimension, the total data samples are lesser. The connections are pruned using a post-process to ensure the affinity graph connectivity with respect to the coefficient matrix for reducing the unused or incorrect connections.

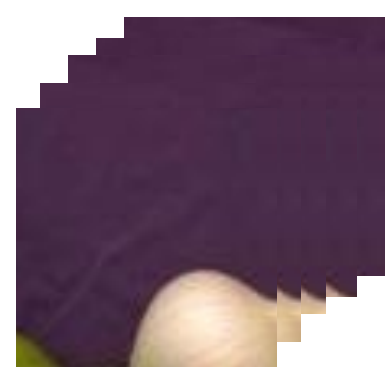

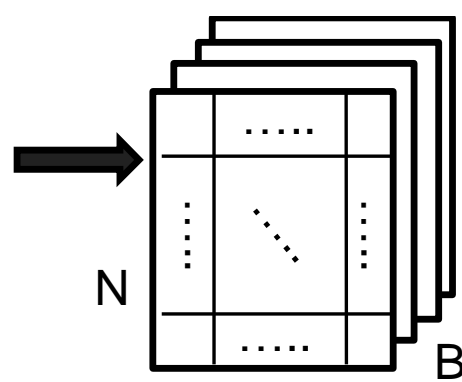

$\mathrm{M}$

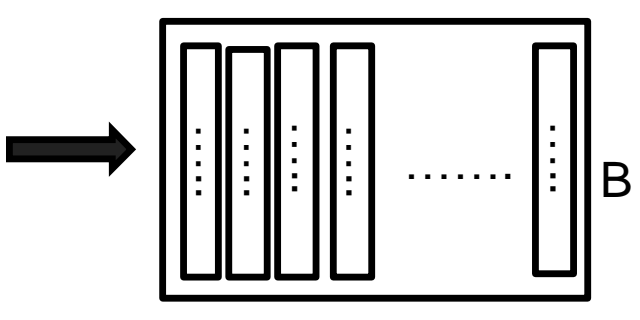

$\mathrm{M} \times \mathrm{N}$

Fig. 2. Three Stage Image Transform Process

Figure 2 represents the three stage image transform process in which the original HSI image is converted to a 3-D cube. Further, the pixels are represented as the column of a twodimensional matrix. $\mathrm{B}$ represents the bands, $\mathrm{N}$ is the rows and $\mathrm{M}$ is the total columns in the 3$\mathrm{D}$ cube. For the sake of clustering, compression of $3 \mathrm{D}$ cube into $2 \mathrm{D}$ cube is done. The 
coefficient is reserved by defining the close neighbours for every sample in the post-process strategy. Neighbours and common neighbour definitions must be introduced to develop close neighbours in a precise manner. It is essential to understand the need for considering specific number of samples along with close and common neighbours. In a subspace, we consider two samples with non-zero coefficients. Considering the data matrix $\mathrm{D} \in \mathfrak{R}^{\mathrm{ds} \mathrm{X}}$, a representation matrix $\mathrm{R}$ is obtained using the traditional subspace clustering scheme. Here, post-processing is performed where the inter-subspace samples are tested for weak correlation and they are pruned. Further, finding close neighbours (FCN) algorithm is used for obtaining a neighbour ranking list $\mathrm{N}$, after reserving the first few layers, for each sample.

Clustering accuracy is significantly affected by the total layers reserved. Reduction in clustering accuracy and overly pruning of the representation matrix is observed if this value is too low. The failure of post-processing strategy is declared if it does not work. For the multiple number of reserved layers, computation of clustering accuracy is performed and total reserved layers are selected in correspondence to the highest clustering accuracy. Spectral clustering is performed in the final stage. The pruning strategy based on close neighbour (PSCN) process comprises of several parameters. However, in order to attain the clustering results, total clusters and data matrix alone are required. Sparsity requirements are satisfied with the proposed pruning technology. In a poorly connected subspace, probability reduction and intra-subspace connection strengthening is performed by employing common neighbours. The proposed model can resolve the trade-off between connectivity and sparsity in an efficient manner.

\section{Results and Discussion}

For the purpose of verification and validation, the experiment is categorised into two segments. Various perspectives are considered for verifying the effectiveness of the proposed model while comparing it with the existing SSC technique. COIL-20, a traditional image dataset is used for the purpose of experimentation. It consists of images from 20 subjects. 72 different angles are used for capturing the pictures of each subject by the camera. 1440 pictures size $128 \times 128$ of are available in the dataset in total. In earth observation system 
based on IoT, the efficiency of the proposed model is verified by comparing with HIS datasets like Pavia University, Salinas, Kennedy Space Centre (KSC) and Indian Pines. The universality of the proposed model is verified by combining it with various algorithms.

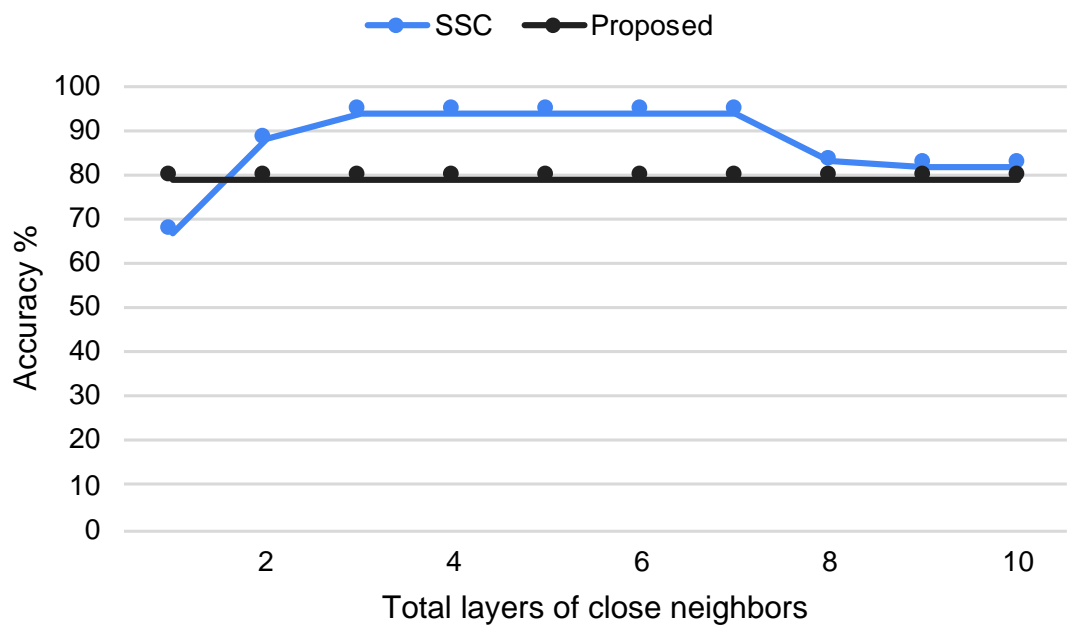

Fig. 3. Total layers of close neighbours vs. Accuracy

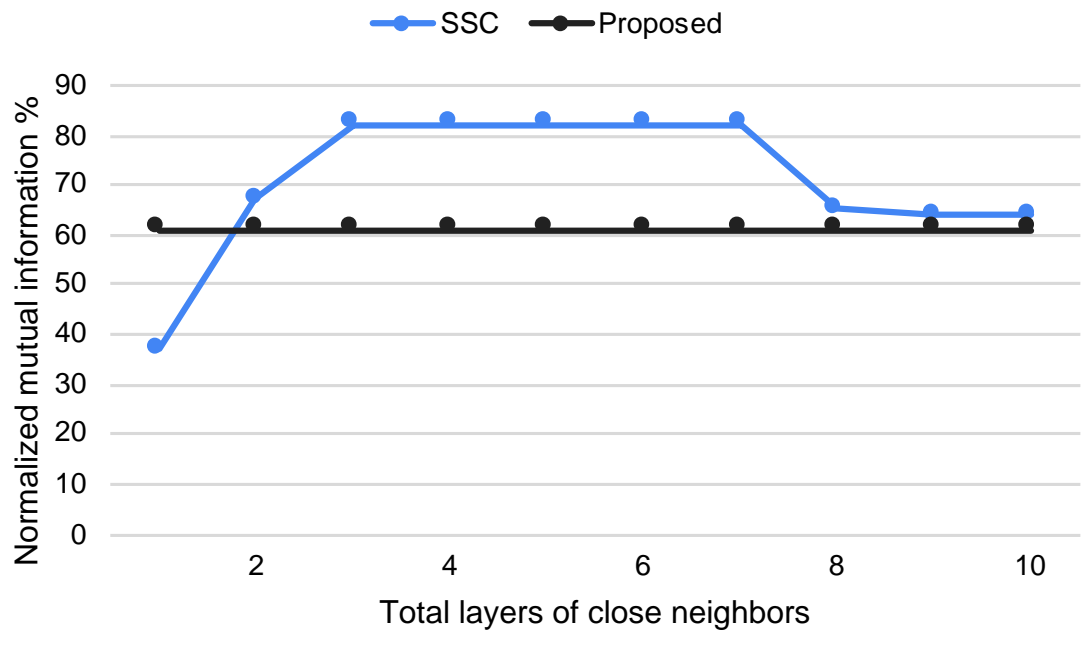

Fig. 4. Total layers of close neighbours vs. Normalized mutual information 
The Indian pines scene obtained from the AVIRIS sensor has images of $145 \times 145$ pixels with a spectral band of 224. From the complete image, three different image blocks are selected with 2400, 1500 and 600 pixels corresponding with 6,5 and 3 classes respectively. $512 \times 614$ pixels and 176 bands KSC data is also used for the verification purpose. Large number of samples are used for verifying the performance of the model. Here, image blocks concluding with 4000 and 1500 are considered as the subsets in which 5 and 3 classes are observed respectively. From the Californian Salinas Valley, the Salinas scene images are gathered by the AVIRIS sensor of 224 band. The images are of 512x217 pixels per band. Image blocks concluding with 2500, 2000 and 1500 are considered in this HSI. 9, 5 and 3 classes of image data are selected for performing the analysis of the proposed model. Figure 3 and 4 represents the comparison of the total number of layers of close neighbours to the accuracy and normalized mutual information.

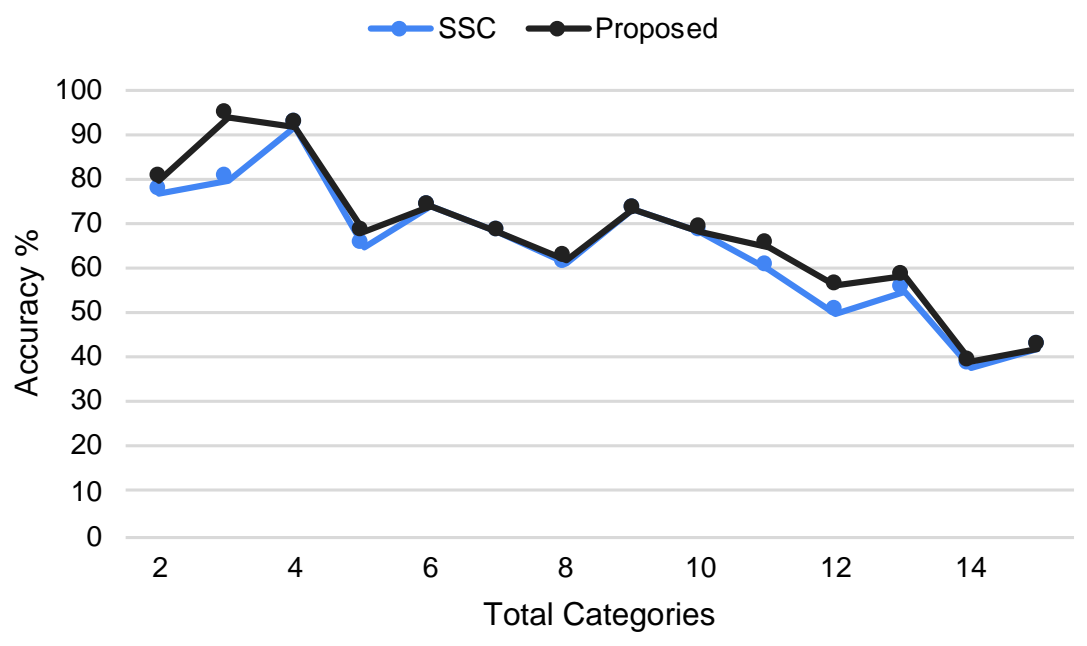

Fig. 5. Total categories vs. Accuracy 


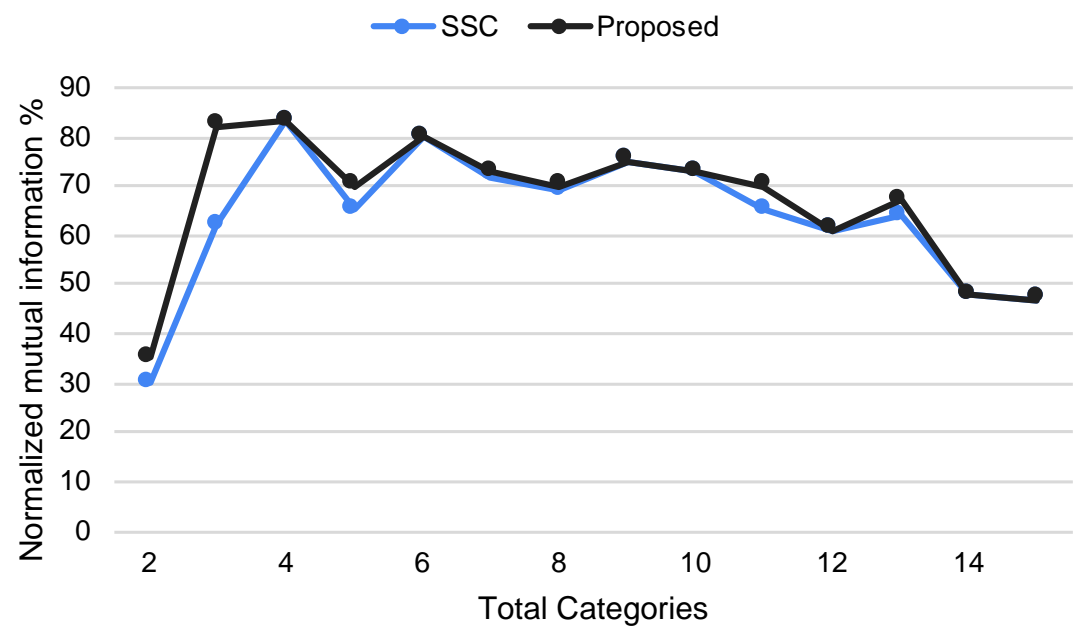

Fig. 6. Total categories vs. Normalized mutual information

Twenty categories are used of which various categories are selected on a random basis. 75 pictures are present in each category, where the number of categories is less than 15 . Figure 5 and 6 represents the variation in accuracy and normalized mutual information with the change in the number of categories. The SSC and PSCN comparison is represented in the graph shown in figure 5 and 6 for analysis of clustering performance. From the results, it is evident that the proposed model outperforms the conventional SSC model despite the variation in total categories. When a relatively smaller number of categories is observed a prominent performance boost is obtained during clustering. The Pavia University based images collected by the ROSIS sensors in northern Italy, during a flight consists of images of $610 \times 610$ pixels with 103 spectral bands. These images are also extracted in the form of three blocks with a varied number of pixels and multiple subjects. Conversion of the image into 3-D cube from the HSI dataset enables further compression into 2-D cube for the purpose of clustering. The data sample is observed as a pixel. The pixels are clustered into the same class with the help of subspace clustering. 


\section{Conclusion}

In this paper, Subspace clustering is employed in edge servers by deploying AI models for prompt reply and cost reduction. At the representation coefficient matrix, the useless and incorrect connections are pruned by means of PSCN, a post-process strategy has been proposed to enhance the clustering performance and reduce the noise effects. Stronger connections are observed at the sample's close neighbours that have larger coefficients and common neighbours. A sparse matrix may be achieved by guaranteeing the intra-subspace connections on reversing the coefficients. Four different HSI data are compared to observe the performance of PSCN along with the clustering algorithms like LRR, SMR, SSCOMP and SSC. In IoT based earth observation system, the proposed method is applicable and it exhibits an improved performance rather than the existing models. The accuracy of sensor based information has been obtained, when HSI increases with clustering by using PSCN. Future work may implement non-dominant sorting to confirm the number of layers that must be reserved directly after clustering.

\section{References}

[1] Yan, F., Wang, X. D., Zeng, Z. Q., \& Hong, C. Q. (2020). Adaptive multi-view subspace clustering for high-dimensional data. Pattern Recognition Letters, 130, 299305.

[2] Sivaganesan, D. (2021). A Data Driven Trust Mechanism Based on Blockchain in IoT Sensor Networks for Detection and Mitigation of Attacks. Journal of trends in Computer Science and Smart technology (TCSST), 3(01), 59-69.

[3] Agarwal, P., \& Mehta, S. (2019). Subspace clustering of high dimensional data using differential evolution. In Nature-inspired algorithms for big data frameworks (pp. 4774). IGI Global.

[4] Chen, J. I. Z., \& Chang, J. T. (2020). Route Choice Behaviour Modeling using IoT Integrated Artificial Intelligence. Journal of Artificial Intelligence, 2(04), 232-237. 
[5] Raj, J. S. (2021). Optimized Mobile Edge Computing Framework for IoT based Medical Sensor Network Nodes. Journal of Ubiquitous Computing and Communication Technologies (UCCT), 3(01), 33-42.

[6] Zhang, G. Y., Zhou, Y. R., He, X. Y., Wang, C. D., \& Huang, D. (2020). One-step kernel multi-view subspace clustering. Knowledge-Based Systems, 189, 105126.

[7] Sungheetha, Akey, and Rajesh Sharma. "3D Image Processing using Machine Learning based Input Processing for Man-Machine Interaction." Journal of Innovative Image Processing (JIIP) 3, no. 01 (2021): 1-6.

[8] Lakshmi, B. J., Madhuri, K. B., \& Shashi, M. (2017). An efficient algorithm for density based subspace clustering with dynamic parameter setting. International Journal of Information Technology and Computer Science, 9(6), 27-33.

[9] Suma, V. (2021). Wearable IoT based Distributed Framework for Ubiquitous Computing. Journal of Ubiquitous Computing and Communication Technologies (UCCT), 3(01), 23-32.

[10] Wang, X., Lei, Z., Guo, X., Zhang, C., Shi, H., \& Li, S. Z. (2019). Multi-view subspace clustering with intactness-aware similarity. Pattern Recognition, 88, 50-63.

[11] Ranganathan, G. "A Study to Find Facts Behind Preprocessing on Deep Learning Algorithms." Journal of Innovative Image Processing (JIIP) 3, no. 01 (2021): 66-74.

[12] Long, Z. Z., Xu, G., Du, J., Zhu, H., Yan, T., \& Yu, Y. F. (2021). Flexible Subspace Clustering: A Joint Feature Selection and K-Means Clustering Framework. Big Data Research, 23, 100170.

[13] Dhaya, R. "Analysis of Adaptive Image Retrieval by Transition Kalman Filter Approach based on Intensity Parameter." Journal of Innovative Image Processing (JIIP) 3, no. 01 (2021): 7-20.

[14] Wang, X. D., Chen, R. C., Yan, F., Zeng, Z. Q., \& Hong, C. Q. (2019). Fast adaptive k-means subspace clustering for high-dimensional data. IEEE Access, 7, 42639-42651.

[15] Dutta, Sayantan, and Ayan Banerjee. "Highly Precise Modified Blue Whale Method Framed by Blending Bat and Local Search Algorithm for the Optimality of 
Image Fusion Algorithm." Journal of Soft Computing Paradigm (JSCP) 2, no. 04 (2020): 195-208.

[16] Zeng, M., Cai, Y., Cai, Z., Liu, X., Hu, P., \& Ku, J. (2019). Unsupervised hyperspectral image band selection based on deep subspace clustering. IEEE Geoscience and Remote Sensing Letters, 16(12), 1889-1893.

[17] Haoxiang, Wang, and S. Smys. "Overview of Configuring Adaptive Activation Functions for Deep Neural Networks-A Comparative Study." Journal of Ubiquitous Computing and Communication Technologies (UCCT) 3, no. 01 (2021): $10-22$.

[18] Lakshmi, B. J., Madhuri, K. B., \& Shashi, M. (2017). An efficient algorithm for density based subspace clustering with dynamic parameter setting. International Journal of Information Technology and Computer Science, 9(6), 27-33.

[19] Lai, Kong-Long, and Joy Iong Zong Chen. "Development of Smart Cities with Fog Computing and Internet of Things." Journal of Ubiquitous Computing and Communication Technologies (UCCT) 3, no. 01 (2021): 52-60.

[20] Zhu, P., Zhu, W., Hu, Q., Zhang, C., \& Zuo, W. (2017). Subspace clustering guided unsupervised feature selection. Pattern Recognition, 66, 364-374.

[21] Lina, S. X., Zhongb, G., \& Shu, T. (2020). Joint Featurewise Weighting and Lobal Structure Learning for Multi-view Subspace Clustering. arXiv preprint arXiv:2007.12829.

\section{Authors Biography}

Bindhu V, received the B.E. degree in Electronics and Communication Engineering from Bharathiar University, Coimbatore, in 2002, M.E. degree in Applied Electronics from Anna University, Chennai, in 2007, and Ph.D. degree from Anna University, Chennai, in 2014. She has 10 years of teaching experience and 5 years of research experience. Currently, she is Professor at PPG Institute of Technology, Coimbatore. Her area of interest includes signal processing and VLSI design. 
G. Ranganathan, Professor and Head, in the Department of Electronics and Communication Engineering, Gnanamani College of Technology, Namakkal, India.. He has done his PhD in the Faculty of Information and Communication Engineering from Anna University, Chennai in the year 2013. His research thesis was in the area of BioMedical Signal Processing. He has a total of $29+$ years of experience both in industry, teaching and research. He has guided several project works for many UG and PG Students in the areas of BioMedical Signal Processing. He has published more than 35 research papers in International and National Journals and Conferences. He has also co-authored many books in electrical and electronics subjects. He has served as Referee for many reputed International Journals published by Elsevier, Springer, Taylor and Francis, etc. He has membership in various professional bodies like ISTE, IAENG etc., and has actively involved himself in organizing various international and national level conferences, symposiums, seminars etc. 